\title{
Seroprevalencia de Brucella canis en perros de un refugio para animales de compañía en Bogotá, Colombia
}

Astrid-Jullieth Laverde1, Daniela Restrepo-Botero', Diego Hernández-Pulido', José Luis Rodríguez-Bautista ${ }^{2}$, Isabel-Sofía Sandoval ${ }^{1}$

${ }^{1}$ Facultad de Medicina Veterinaria, Fundación Universitaria Agraria de Colombia, Bogotá, D.C., Colombia

${ }^{2}$ Programa de Pós-Graduação em Ciências Veterinárias, Universidade Federal Rural do Rio de Janeiro, Seropédica, Rio de Janeiro, Brasil

Introducción. El riesgo de infección con Brucella canis en humanos y perros aumenta con la exposición constante a perros portadores asintomáticos. En Colombia hay evidencia de infección con $B$. canis en personas que conviven con perros. Una preocupación adicional en Bogotá es la falta de información actualizada sobre la prevalencia de la infección en perros destinados a programas de adopción.

Objetivo. Establecer la seroprevalencia de la infección por B. canis en perros de un refugio para animales de compañía destinados a la adopción en Bogotá.

Materiales y métodos. Se hizo un estudio descriptivo de corte transversal en un refugio para animales de Bogotá. Se detectaron anticuerpos contra B. canis en el suero de 51 perros (28 hembras y 23 machos) mediante una prueba inmunocromatográfica de flujo lateral. Asimismo, los individuos positivos se analizaron con PCR para la detección del ADN de Brucella spp.

Resultado. La seroprevalencia de B. canis fue del 1,96 \% (1/51). El perro seropositivo correspondió a una hembra asintomática de tres años de edad en la cual no se detectó ADN bacteriano en sangre mediante la PCR.

Conclusiones. La seroprevalencia representada por un solo perro con IgG anti-B. canis puede considerarse un riesgo potencial para las poblaciones de perros y humanos, ya que

Recibido: 06/02/2020

Aceptado: $27 / 11 / 2020$

Publicado: 27/11/2020

Citación:

Laverde A-J, Restrepo-Botero D, HernándezPulido D, Rodríguez-Bautista JL, Sandoval I-S. Seroprevalencia de Brucella canis en perros de un refugio para animales de compañía en Bogotá, Colombia. Biomédica. 2021;41:260-70. https://doi.org/10.7705/biomedica.5409

\section{Correspondencia:}

Isabel Sofía Sandoval, Facultad de Medicina Veterinaria, Fundación Universitaria Agraria de Colombia, Calle 170 No 54A - 10, Bogotá, D.C., Colombia

Teléfono: (571) 667 1515, extensión 222

isabelssb@gmail.com y sandoval.isabel@uniagraria. edu.co

Contribución de los autores:

Astrid-Jullieth Laverde y Daniela Restrepo-Botero: toma y procesamiento de muestras y redacción del manuscrito

Diego Hernández-Pulido: coordinación de la selección del refugio y de la toma de muestras, y revisión del manuscrito

José Luis Rodríguez-Bautista: diseño del estudio y redacción del manuscrito

Isabel-Sofía Sandoval: diseño del estudio, toma

y procesamiento de muestras y redacción del

manuscrito

Financiación:

Este trabajo se llevó a cabo con el apoyo financiero de la Vicerrectoría de Investigaciones de la Fundación Universitaria Agraria de Colombia.

Conflicto de intereses:

Los autores declaran no tener ningún conflicto de intereses para la publicación de este artículo. podría tratarse de un animal con infección persistente capaz de diseminar la bacteria.

Palabras clave: brucelosis; perros; zoonosis; cromatografía de afinidad; salud pública.

\section{Seroprevalence of Brucella canis in canines from a dog shelter in Bogotá, Colombia}

Introduction: The risk of Brucella canis infection in humans and dogs has increased due to the permanent exposure to asymptomatic carrier dogs. In Colombia, there is evidence of $B$. canis infection in humans living with dogs. In the case of Bogotá, an additional concern is the lack of updated information related to the prevalence of the infection in dogs.

Objective: To determine the seroprevalence of infection by $B$. canis in dogs intended for adoption programs in Bogotá.

Materials and methods: By means of a descriptive cross-sectional study carried out in a dog shelter in Bogotá, anti-B. canis IgG antibodies were detected in the serum from 51 dogs (28 females and 23 males) using a lateral-flow immunochromatographic test. Additionally, seropositive animals were analyzed with PCR to detect Brucella spp DNA.

Results: Brucella canis seroprevalence was $1.96 \%$ (1/51). The seropositive dog was an asymptomatic three-year-old she-dog in which no bacteria DNA was detected in the blood through PCR.

Conclusions: The seroprevalence determined in this study represented by a single dog with anti-B. canis IgG can be considered a potential risk both for canine and human populations since this single dog could have a persistent infection capable of spreading the bacteria.

Keywords: Brucellosis, dogs; zoonoses; chromatography, affinity; public health.

La bacteria Brucella canis es considerada el principal agente causal de la brucelosis canina (1-3), aunque otras especies del género, como $B$. abortus, $B$. suis y $B$. melitensis, pueden infectar de forma transitoria a los perros (4). El cuadro clínico en el perro es variable y se encuentran desde casos asintomáticos $(1,5,6)$, hasta abortos, orquitis, epididimitis, prostatitis $(2,7)$, discoespondilitis (8), endoftalmitis (9) o linfadenomegalia (10). La transmisión a los animales vulnerables suele darse por medio del coito, los aerosoles o por contacto directo de mucosas o piel lesionada con material contaminado con el agente patógeno $(2,11)$. 
Se sabe que los fluidos vaginales posteriores a un aborto y el semen de animales infectados podrían constituir algunas de las fuentes más importantes de contagio para otros perros y para las personas, dada la elevada concentración de bacterias presentes en estas secreciones $(11,12)$. Sin embargo, como $B$. canis puede persistir en secreciones vaginales no asociadas con el período de celo (estro) y en órganos diferentes a los reproductivos, el agente patógeno puede también diseminarse de manera intermitente y en bajas concentraciones en orina, saliva y secreciones nasales, por lo que la castración de hembras y machos no elimina totalmente el riesgo de contagio $(2,11-14)$.

El tratamiento antibiótico para la brucelosis canina no siempre es efectivo para erradicar la bacteria, por lo que pueden presentarse picos recurrentes de bacteriemia después de finalizado el tratamiento $(15,16)$, lo cual podría aumentar el riesgo de diseminación bacteriana a individuos vulnerables.

El contacto directo con semen, secreciones, membranas fetales y cachorros abortados de hembras infectadas, sigue siendo la principal ruta de transmisión al ser humano $(17,18)$. La infección también puede ocurrir por medio de fómites y se sabe que la bacteria puede sobrevivir por largos períodos en el suelo y el agua contaminados $(11,19,20)$.

Es muy probable que la infección por $B$. canis en los seres humanos se encuentre subdiagnosticada debido a la sintomatología inespecífica $(3,4,13)$, y a que la bacteria no se puede identificar mediante las pruebas serológicas empleadas de rutina para el diagnóstico de otras especies del género (13). Aunque todavía se consideran poco frecuentes, un número creciente de estudios en la última década ha documentado infecciones con $B$. canis como causa de endocarditis, peritonitis y otros cuadros clínicos (4). En Colombia, la bacteria se aisló por primera vez en el 2009 en la sangre de una mujer asintomática propietaria de un criadero, cuyos perros presentaban problemas reproductivos asociados con la infección por $B$. canis (21). Posteriormente, Sánchez-Jiménez, et al., informaron dos casos no publicados de médicos veterinarios seropositivos y con sintomatología sugestiva de brucelosis (22).

Desde el punto de vista zoonótico, quizá lo más relevante de esta bacteria es el riesgo ocupacional que representa para veterinarios, criadores de perros y empleados de refugios o perreras (18). No obstante, es poco el énfasis en el riesgo de transmisión por perros destinados a la adopción. Aunque $B$. canis se considera de bajo potencial zoonótico en comparación con otras especies del mismo género, se ha demostrado que el contacto estrecho con perros infectados aumenta el riesgo de contagio $(13,17,23)$, especialmente en adultos mayores, niños e individuos inmunocomprometidos $(2,24)$. La zoonosis por $B$. canis parece ser un problema emergente en residentes de barrios urbanos marginales y de bajo nivel socioeconómico, en los que sean numerosos los perros callejeros (4).

La seroprevalencia de $B$. canis en algunos albergues de caninos en Colombia varía entre $0 \%$ en Envigado (25) y Bucaramanga (26), y 6,78 \% en Medellín (27). No hay publicaciones recientes de estudios en refugios de Bogotá. En el ámbito mundial, los resultados también varían, pues se han encontrado prevalencias que oscilan entre 0,4 y $20,9 \%$ en perros de refugios o domésticos $(10,11,24,28-32)$.

En el diagnóstico serológico y como prueba de tamización, puede emplearse la detección cualitativa de anticuerpos lgG anti- $B$. canis mediante inmunocromatografía rápida (Anigen ${ }^{\mathrm{TM}}$ ), con $93,0 \%$ de sensibilidad 
y $100,0 \%$ de especificidad (33); posteriormente, es posible recurrir a pruebas complementarias como la de aglutinación rápida en placa con 2 $\beta$-mercaptoetanol (PARP-2ME) (19).

El cultivo de sangre se considera la prueba de referencia, pero tiene poca sensibilidad y los resultados se demoran, por lo que la reacción en cadena de la polimerasa (PCR) puede considerarse una prueba de ayuda diagnóstica complementaria en el seguimiento o vigilancia de la enfermedad, pues detecta pequeñas cantidades del ADN bacteriano. La amplificación de un fragmento del gen bp26, que codifica para la proteína periplasmática e inmunodominante BP26 de Brucella spp. (34), con una sensibilidad de 92,6\% y una especificidad de $90,0 \%$ frente al hemocultivo, puede ser la prueba de elección o complementaria para el diagnóstico de la infección por B. canis (35).

Teniendo en cuenta la escasa información epidemiológica sobre esta zoonosis, el objetivo de este estudio transversal fue determinar la seroprevalencia de $B$. canis en perros de un refugio para animales de compañía ubicado en Bogotá.

\section{Materiales y métodos}

Se hizo un estudio descriptivo de corte transversal en un refugio de animales ubicado en Bogotá, que funciona como albergue transitorio de perros abandonados en la zona urbana, donde permanecen hasta su adopción, muerte o eutanasia.

\section{Tamaño de la muestra}

Para el cálculo del tamaño de la muestra (n) en una población de 145 perros, se empleó el módulo "Tamaño de muestra: detectar enfermedad (muestreo aleatorio y diagnóstico perfecto", de la plataforma Working in Epidemiology (WinEpi) (http://www.winepi.net/f101.php). La prevalencia mínima esperada se fijó en 5,0\% con un nivel de confianza del 95,0\% y se obtuvo un número de 51 individuos seleccionados mediante muestreo aleatorio por conveniencia.

\section{Toma de muestras}

Después de obtener el consentimiento informado de la propietaria del refugio, se tomaron muestras de sangre por punción de las venas yugular o cefálica de los animales, en tubos al vacío sin anticoagulante (Vacutainer system $^{\mathrm{TM}}$ ); las muestras se rotularon con la respectiva identificación del animal y se refrigeraron hasta su envío al laboratorio. Los sueros se obtuvieron por centrifugación a $1.008 \mathrm{~g}$ durante 5 minutos y, posteriormente, se congelaron a $-20^{\circ} \mathrm{C}$ en tubos de polipropileno de 1,5 ml (Eppendorf Tubes $^{\mathrm{TM}}$ ) hasta el momento de su procesamiento.

\section{Diagnóstico serológico}

Los anticuerpos (IgG) contra B. canis se detectaron usando un inmunoensayo de cromatografía de flujo lateral (Bionote, Inc., kit de inmunocromatografía Prueba Rápida Anigen ${ }^{\mathrm{TM}}$, Gyeonggi-do, Corea) siguiendo las especificaciones del fabricante. Brevemente, se adicionaron $10 \mu \mathrm{l}$ de suero al pozo de la muestra, a continuación, se agregaron 2 gotas (aproximadamente $80 \mu \mathrm{l}$ ) de solución tampón diluyente y se interpretaron después de 20 minutos de incubación a temperatura ambiente. La presencia de dos bandas de color (banda $T$ y banda $C$ ) en la ventana de resultados, se interpretó como un resultado positivo de anticuerpos contra B. canis (33). 
Las muestras positivas por inmunocromatografía se remitieron a un laboratorio externo de referencia para la detección de IgG mediante la prueba de aglutinación rápida en placa con $2 \beta$-mercaptoetanol (PARP-2ME) (19). El procedimiento empleado por el laboratorio fue el siguiente: se mezclaron a partes iguales el 2-mercaptoetanol y el suero problema, y la mezcla se expuso al antígeno de la cepa $\mathrm{M}$ de $B$. canis. La aglutinación indicó que el suero era positivo para IgG contra $B$. canis.

\section{Diagnóstico molecular}

Las muestras de los animales seropositivos se analizaron por PCR para Brucella spp. en ADN extraído a partir de sangre completa previamente recolectada en tubos $B D$ Vacutainer ${ }^{\mathrm{TM}}$ con citrato de sodio y los oligonucleótidos BMEI0535F (5'-GCGCATTCTTCGGTTATGAA-3') y BMEI10536R

(5'-CGCAGGCGAAAACAGCTATAA-3'), reportados por Sánchez-Jiménez, et al., los cuales amplifican un fragmento de $451 \mathrm{pb}$ del gen bp26 de Brucella (35).

Las condiciones usadas fueron las mismas empleadas por SánchezJiménez, et al., y Olivera, et al. $(35,36)$ : la solución de reacción contenía $3 \mu \mathrm{l}$ de $\mathrm{MgCl}_{2}$ (3 mM, Fermentas, Foster City, CA, USA), 2,5 $\mu$ l de solución tampón Tris$\mathrm{HCl} 10 \mathrm{mM}$; tritón 100X y KCl con pH de 8,8 (Fermentas, Foster City, CA, USA), $0,5 \mu \mathrm{l}$ de dNTP $(10 \mathrm{mM}), 3 \mu \mathrm{l}$ de plantilla de ADN ( $1 \mathrm{ng} / \mu \mathrm{l}), 0,625 \mu \mathrm{l}$ de cada oligonucleótido $(0,25 \mu \mathrm{M}), 0,2 \mu \mathrm{l}$ de ADN de polimerasa Taq $(5 \mathrm{Ul} / \mu \mathrm{l}$, Fermentas Taq DNA polimerasa recombinante, Foster City, CA, USA) y se empleó agua destilada para completar un volumen final de reacción de $25 \mu \mathrm{l}$ (35).

La amplificación se hizo en un termociclador PTC 100 ${ }^{\text {TM }}$ (Perkin-Elmer Inc., San José, CA, USA) en un total de 25 ciclos de desnaturalización durante un minuto a $95{ }^{\circ} \mathrm{C}$, alineamiento de los oligonucleótidos a $64^{\circ} \mathrm{C}$ durante 45 segundos, y extensión de la cadena a $72{ }^{\circ} \mathrm{C}$ durante tres minutos. Finalizados los 25 ciclos, se realizó una extensión final a $72{ }^{\circ} \mathrm{C}$ durante seis minutos (36). Los productos de la PCR se visualizaron en una electroforesis en gel de agarosa al $1 \%$ con $0,5 \mu \mathrm{g} / \mathrm{ml}$ de bromuro de etidio y la ayuda de un transiluminador ultravioleta (Transilluminator Mini Benchtop Model M-10E ${ }^{\mathrm{TM}}$, UVP, Upland, CA, USA). Se utilizó el marcador de peso molecular de 50 pb (50 a 1.000 pb), Generuler ${ }^{\mathrm{TM}}$ (Fermentas Inc., Burlington, Canadá) (35).

Como control positivo de la prueba, se utilizó el ADN extraído de la cepa de $B$. canis menos mucoide (M-) empleada en la preparación del antígeno para pruebas serológicas. Como control negativo, se emplearon todos los reactivos de la PCR, excepto el ADN (35).

\section{Análisis de los datos}

Se elaboró una base de datos en formato Excel ${ }^{\mathrm{TM}}$, en la que se registró la información relevante de los perros evaluados: sexo, edad y estado reproductivo (enteros o castrados, sometidos a orquiectomía u ovariohisterectomía). Mediante el inmunoensayo de cromatografía de flujo lateral, se estableció la seroprevalencia para $B$. canis y se comparó por sexo, grupo etario (grupo I: $\leq 1$ año; grupo II: $>1$ año y $<6$ años, y grupo III: $\geq 6$ años) y estado reproductivo (castrados o enteros) en el grupo de estudio.

\section{Consideraciones éticas}

El proyecto fue aprobado por el Comité de Ética y Bioética de la Fundación Universitaria Agraria de Colombia, después de verificar que los protocolos propuestos se ajustaran a las normas éticas para toma de muestras clínicas 
en animales, de conformidad con lo establecido en el Artículo 88 y el literal e) del Artículo 87 de la Resolución 8430 de 1993 (Normas científicas, técnicas y administrativas para la investigación en salud), y los artículos 15 y 16, Capítulo I, Título II de la Ley 576 de 2000 (Código de ética para el ejercicio profesional de la medicina veterinaria, la medicina veterinaria y zootecnia y zootecnia).

\section{Resultados}

Se analizaron los sueros de 51 perros procedentes de un refugio para animales de compañía, de los cuales 28 eran hembras y 23 machos, 44 eran castrados y siete eran enteros. La edad de los animales fluctuaba entre los 4 meses y los 15 años (cuadro 1).

La seroprevalencia para B. canis por inmunocromatografía fue de 1,96. Por otro lado, ninguno de los machos analizados fue seropositivo, en tanto que una hembra (3,6 \% del total de hembras) fue seropositiva, tanto por inmunocromatografía como por PARP-2ME, aunque sin evidencia de $A D N$ bacteriano en la sangre en la PCR (figura 1). En cuanto al estado reproductivo, la hembra con anticuerpos anti- $B$. canis estaba esterilizada, lo que se presenta en el $2,3 \%$ de los animales bajo esta condición. Con respecto a la edad, la serorreacción fue de $0,0,4,3$ y 0,0 \% para los grupos I, II y III, respectivamente (cuadro 1).

Cuadro 1. Prevalencia de anticuerpos contra Brucella canis según, sexo, edad y estado reproductivo de 51 perros de un refugio de mascotas en Bogotá

\begin{tabular}{lccl}
\hline & \multicolumn{4}{c}{ Total (n) } & Seronegativos n (\%) & Seropositivos n (\%) \\
\hline Sexo & & & \\
$\quad$ Macho & 23 & $23(100)$ & $0(0)$ \\
Hembra & 28 & $27(96,4)$ & $1(3,6)$ \\
Edad (años) & & & \\
$\quad$ Grupo I $(\leq 1)$ & 10 & $10(100)$ & $0(0)$ \\
Grupo II $(>1$ y $<6)$ & 23 & $22(95,7)$ & $1(4,3)$ \\
$\quad$ Grupo III $(\geq 6)$ & 18 & $18(100)$ & $0(0)$ \\
Estado reproductivo & & & \\
$\quad$ Castrado & 44 & $43(97,7)$ & $1(2,3)$ \\
$\quad$ Entero & 7 & $7(100)$ & $0(0)$ \\
Total & 51 & $50(98,04)$ & $1(1,96)$ \\
\hline
\end{tabular}

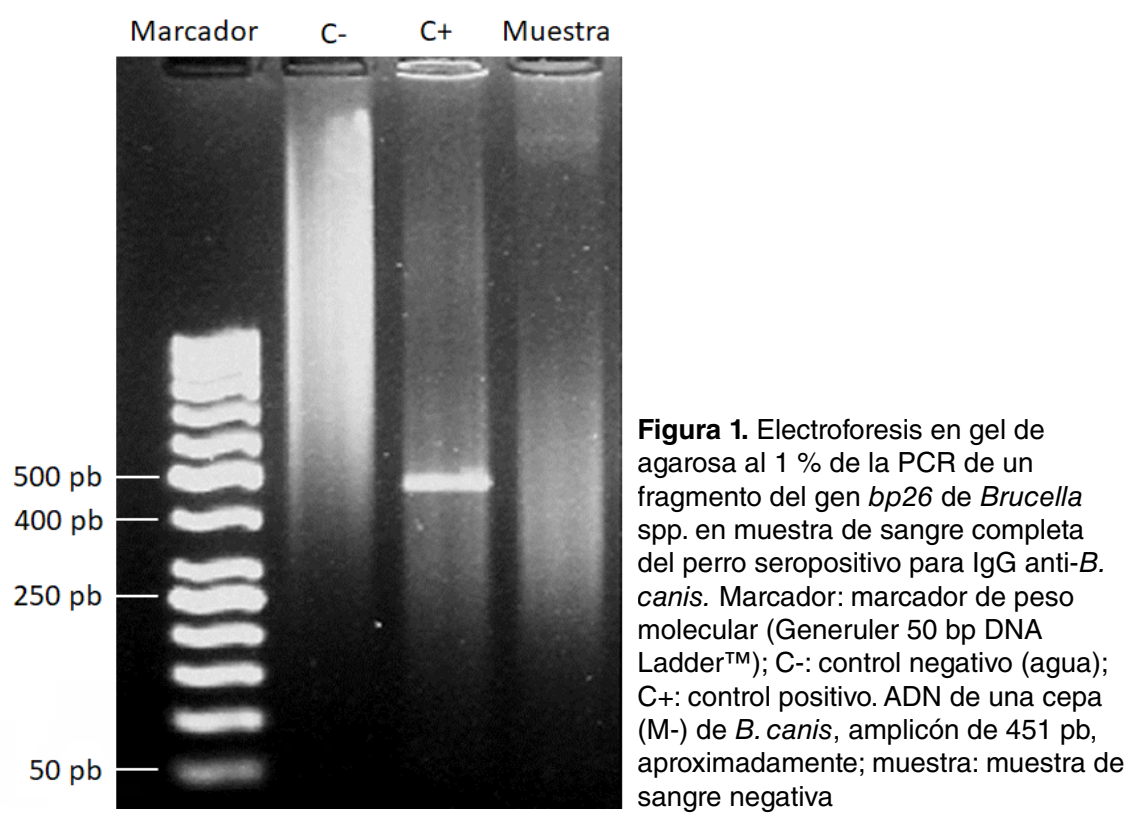




\section{Discusión}

La seroprevalencia de $B$. canis en el inmunoensayo de cromatografía de flujo lateral en los perros del refugio fue del 1,96 \%, correspondiente a una hembra de tres años sin signos clínicos de brucelosis. Esta seroprevalencia difiere de la encontrada en otros estudios en animales de albergues en diferentes regiones de Colombia, y es mayor a la observada en los estudios desarrollados en las ciudades de Envigado (25) y Bucaramanga (26), donde no se encontraron animales seropositivos mediante inmunocromatografía. Por el contrario, la seroprevalencia encontrada en este trabajo fue menor que la reportada en los estudios realizados en animales de albergue en Villavicencio y Medellín, en los que se determinaron valores de 9,3\% (37) y 6,78 \% (27), respectivamente, mediante la prueba de aglutinación rápida en placa con 2 $\beta$-mercaptoetanol (PARP-2ME).

Contrario a esto, la seroprevalencia encontrada en Bogotá fue mayor que la obtenida por Pardo, et al., en un estudio mixto con perros callejeros y domésticos, la cual fue de 1,49 \% empleando la prueba PARP-2ME (38).

Por otro lado, cabe resaltar que la seroprevalencia hallada en este estudio fue menor que la reportada en trabajos previos con perros domésticos en tres ciudades colombianas. En Medellín, fue del 2,76 \% según la inmunocromatografía (39) y del 6,6 \% con la PARP-2ME (40) en perros de asentamientos urbanos de bajo perfil socioeconómico (comunas), y del $11,0 \%$, en perros de criaderos o de clínicas veterinarias según la prueba de PARP-2ME (23). En Montería, la seroprevalencia encontrada con inmunocromatografía en hembras de entornos urbanos fue de $11,0 \%(41)$. También, vale la pena resaltar que la seroprevalencia hallada es mucho menor que la observada por Castillo, et al., (citado por Giraldo, et al.), la cual correspondió al 20,3 \% en una clínica veterinaria universitaria de Bogotá en el $2002(23)$.

Por otro lado, en el ámbito mundial los reportes de prevalencia de B. canis con base en serología o cultivo también se caracterizan por el alto índice de variabilidad, ya que se encuentran valores entre 0,40 y $20,9 \%(10,11,28-32)$.

El amplio rango de prevalencias reportadas en Colombia y otros países podría atribuirse a múltiples factores, entre ellos, el método de muestreo, las características de la población estudiada (2), y la sensibilidad y especificidad de las pruebas de diagnóstico empleadas (42).

Es importante tener en cuenta que la seroconversión contra $B$. canis se inicia con el incremento de lgM hacia la tercera o cuarta semana, lo que se puede extender hasta la semana 12 después de la exposición $(13,43,44)$. Entonces, pueden esperarse falsos negativos cuando las pruebas serológicas se hacen antes de la seroconversión o, en infecciones crónicas, cuando los títulos de anticuerpos circulantes se encuentran por debajo de los niveles de detección de las pruebas serológicas (13,44-46). Por consiguiente, dado que la reacción de los anticuerpos a una infección reciente o leve varía según las situaciones que se presenten después de la exposición, la técnica serológica empleada podría no detectar infecciones recientes o infecciones crónicas que se estuviesen presentando en la población de estudio.

Además de la dinámica de la respuesta humoral a la infección por $B$. canis, es necesario señalar que las características inherentes a toda prueba diagnóstica inciden directamente en el análisis de los resultados. En este 
caso, la poca probabilidad de encontrar falsos positivos (especificidad del 100 $\%$ ) contrasta con la posibilidad de obtener falsos negativos (sensibilidad del $93 \%$ ) (33). Por consiguiente, debe considerarse que la seroprevalencia en la población de estudio puede ser superior a la encontrada.

No se pudo demostrar la presencia de ADN de B. canis con la prueba de PCR en el individuo seropositivo, pese a ser este un método más sensible para determinar la infección que el hemocultivo y la serología (46). La bacteriemia por B. canis tiende a desarrollarse hacia la segunda a cuarta semana de la infección y es de carácter intermitente (45). Durante la bacteriemia, el agente patógeno coloniza los tejidos diana como órganos reproductivos, hígado, bazo y médula ósea $(15,37,38,46-48)$, o puede localizarse en los ganglios linfáticos $(49,50)$. Por ello, es posible obtener PCR positivas sin seroconversión al inicio de la infección (13), o PCR negativas en animales seropositivos que se encuentren en la fase crónica de la infección con localización de la bacteria en órganos blanco (13,46-48), como posiblemente sucedió en este caso.

No se encontró IgG en otros perros en estrecho contacto con la hembra infectada. Además de la sensibilidad de la técnica diagnóstica, puede haber otros factores asociados con la presencia de animales seronegativos en la población de estudio. Aspectos como la castración del $86,3 \%$ de los animales y la consiguiente ausencia de prácticas reproductivas, pudieron contribuir a minimizar el riesgo de contagio $(51,52)$.

El impacto de esta infección animal en la salud de los seres humanos no ha sido completamente caracterizado porque gran parte de la información proviene de reportes de casos o informes de brotes (11,17,53-55), y, además, porque las pruebas serológicas disponibles en el mercado detectan especies de las cepas lisas de Brucella y no anticuerpos contra B. canis (56), lo que ha conducido, finalmente, a que existan pocos estudios de prevalencia. Aunque desde el punto de vista ocupacional se considera que los cuidadores de los perros en los refugios o criaderos, los médicos veterinarios y el personal de laboratorio son los principales grupos de riesgo (18), con la evidencia científica también se destaca como factor de riesgo potencial el tener perros como animales de compañía (11), especialmente para los niños $(55,57)$, las personas inmunocomprometidas $(53,54)$, las de edad avanzada (58) o las mujeres gestantes (59).

El aumento descontrolado de la población canina causa un impacto negativo en la salud pública (60). Los perros callejeros representan un factor de riesgo de infección para las personas y para otros animales $(32,60,61)$. Las enfermedades en perros con tutores responsables son relativamente más fáciles de controlar, en comparación con la población callejera, puesto que esta deambula por la calle y tiene más probabilidades de entrar en contacto con otros perros infectados y luego convertirse en potenciales diseminadores del agente patógeno (32). Por lo tanto, las estrategias de diagnóstico no deberían excluir las poblaciones de perros callejeros, con el fin de determinar la prevalencia e implementar las medidas pertinentes de control de B. canis en estos grupos.

La presencia de perros asintomáticos potencialmente adoptables podría constituir un gran riesgo para la población humana y animal, lo que debe alertar al personal sanitario sobre la importancia de hacer tamizaciones periódicas para detectar la infección en los dos grupos poblacionales. 
Los resultados del presente estudio aportan información actualizada de la seroprevalencia de $B$. canis en un refugio de perros en Bogotá y pueden ser la base para futuras investigaciones de la enfermedad, tanto en perros callejeros como en animales de compañía.

\section{Agradecimientos}

Los autores agradecen al Grupo de Investigación en Ciencias Animales de la Fundacion Universitaria Agraria de Colombia, por apadrinar este proyecto, así como a los propietarios y al personal del refugio.

\section{Referencias}

1. Batinga MC, Dos Santos JC, Lima JT, Bigotto MF, Muner K, Faita T, et al. Comparison of three methods for recovery of Brucella canis DNA from canine blood samples. J Microbiol Methods. 2017;143:26-31. https://doi.org/10.1016/j.mimet.2017.08.019

2. Hensel ME, Negron M, Arenas-Gamboa AM. Brucellosis in dogs and public health risk. Emerg Infect Dis. 2018;24:1401-6. https://doi.org/10.3201/eid2408.171171

3. Pujol M, Borie C, Montoya M, Ferreira A, Vernal R. Brucella canis induces canine CD4+ T cells multi-cytokine Th1/Th17 production via dendritic cell activation. Comp Immunol Microbiol Infect Dis. 2019;62:68-75. https://doi.org/10.1016/i.cimid.2018.11.017

4. Olsen SC, Palmer MV. Advancement of knowledge of Brucella over the past 50 years. Vet Pathol. 2014;51:1076-89. https://doi.org/10.1177/0300985814540545

5. Chacón-Díaz C, Altamirano-Silva P, González-Espinoza G, Medina MC, Alfaro-Alarcón A, Bouza-Mora L, et al. Brucella canis is an intracellular pathogen that induces a lower proinflammatory response than smooth zoonotic counterparts. Infect Immun. 2015;83:486170. https://doi.org/10.1128/IAl.00995-15

6. Gyuranecz M, Szeredi L, Rónai Z, Dénes B, Dencso L, Dán Á, et al. Detection of Brucella canis-induced reproductive diseases in a Kennel. J Vet Diagnostic Investig. 2011;23:143-7. https://doi.org/10.1177/104063871102300127

7. Byndloss MX, Tsolis RM. Brucella spp. virulence factors and immunity. Annu Rev Anim Biosci. 2016;4:111-27. https://doi.org/10.1146/annurev-animal-021815-111326

8. Forbes JN, Frederick SW, Savage MY, Cross AR. Brucella canis sacroiliitis and discospondylitis in a dog. Can Vet J. 2019;60:1301-4.

9. Ledbetter EC, Landry MP, Stokol T, Kern TJ, Messick JB. Brucella canis endophthalmitis in 3 dogs: Clinical features, diagnosis, and treatment. Vet Ophthalmol. 2009;12:183-91. https://doi.org/10.1111/j.1463-5224.2009.00690.x

10. Keid LB, Chiebao DP, Batinga MC, Faita T, Diniz JA, Oliveira TM, et al. Brucella canis infection in dogs from commercial breeding kennels in Brazil. Transbound Emerg Dis. 2017;64:691-7. https://doi.org/10.1111/tbed.12632

11. Johnson CA, Carter TD, Dunn JR, Baer SR, Schalow MM, Bellay YM, et al. Investigation and characterization of Brucella canis infections in pet-quality dogs and associated human exposures during a 2007-2016 outbreak in Michigan. J Am Vet Med Assoc. 2018;253:322-36. https://doi.org/10.2460/javma.253.3.322

12. Carmichael LE, Joubert JC. Transmission of Brucella canis by contact exposure. Cornell Vet. 1988;78:63-73.

13. Kauffman LK, Petersen CA. Canine brucellosis: Old foe and reemerging scourge. Vet Clin North Am Small Anim Pract. 2019;49:763-79. https://doi.org/10.1016/j.cvsm.2019.02.013

14. De Souza TD, De Carvalho TF, Mol JP, Lopes JV, Silva MF, Da Paixão TA, et al. Tissue distribution and cell tropism of Brucella canis in naturally infected canine foetuses and neonates. Sci Rep. 2018;8:7203-12. https://doi.org/10.1038/s41598-018-25651-X

15. Wanke MM, Delpino MV, Baldi PC. Use of enrofloxacin in the treatment of canine brucellosis in a dog kennel (clinical trial). Theriogenology. 2006;66:1573-8. https://doi.org/10.1016/j.theriogenology

16. Boeri EJ, Escobar GI, Ayala SM, Hasan D, Lucero NE. Tracking dogs infected with Brucella canis after antibiotic treatment. Arch Anim Husb Dairy Sci. 2018;1:1-4. https://doi.org/10.33552/AAHDS.2018.01.000505 
17. Lucero NE, Corazza R, Almuzara MN, Reynes E, Escobar GI, Boeri E, et al. Human Brucella canis outbreak linked to infection in dogs. Epidemiol Infect. 2010;138:280-5. https://doi.org/10.1017/S0950268809990525

18. Krueger WS, Lucero NE, Brower A, Heil GL, Gray GC. Evidence for unapparent Brucella canis infections among adults with occupational exposure to dogs. Zoonoses Public Health. 2014;61:509-18. https://doi.org/10.1111/zph.12102

19. Castrillón-Salazar L, Giraldo-Echeverri CA, Sánchez-Jiménez MM, Olivera-Angel M. Factores asociados con la seropositividad a Brucella canis en criaderos caninos de dos regiones de Antioquia, Colombia. Cad Saúde Pública. 2013;29:1955-73. https://doi.org/10.1590/0102-311X00133013

20. Makloski CL. Canine brucellosis management. Vet Clin North Am Small Anim Pract. 2011;41:1209-19. https://doi.org/10.1016/j.cvsm.2011.08.001

21. Olivera M, Di-Lorenzo C. Aislamiento de Brucella canis en un humano conviviente con caninos infectados. Informe de un caso. Colomb Med. 2009;40:218-20.

22. Sánchez-Jiménez MM, Giraldo-Echeverri CA, Olivera-Angel M. Infección por Brucella canis en humanos: propuesta de un modelo teórico de infección a través de la ruta oral. Infectio. 2013;17:193-200. https://doi.org/10.1016/S0123-9392(13)70731-8

23. Giraldo-Echeverri CA, Ruiz-Cortés ZT, Olivera-Ángel M. Brucella canis en Medellín (Colombia), un problema actual. Revista UDCA Actualidad \& Divulgación Científica. 2009;12:51-5.

24. Whitten TV, Brayshaw G, Patnayak D, Álvarez J, Larson CM, Root Kustritz M, et al. Seroprevalence of Brucella canis antibodies in dogs entering a Minnesota humane society, Minnesota, 2016-2017. Prev Vet Med. 2019;168:90-4. https://doi.org/10.1016/j. prevetmed.2019.04.015

25. Agudelo P, Molina V, Arias V, Madrigal E. Estudio serológico de brucelosis canina en dos albergues del municipio de Envigado, Colombia (2011). Revista de la Facultad de Medicina Veterinaria y de Zootecnia. 2014;61:134-41. https://doi.org/10.15446/rfmvZ.v61n2.44676

26. Uribe R, Delgado K. Determinación de la presencia de Brucella canis en caninos de dos refugios de la ciudad de Bucaramanga en 2012. CES Medicina Veterinaria y Zootecnia. 2013;8:95-103.

27. Ruiz-Buitrago JD, Giraldo-Echeverri CA, López LV, Chica JF. Seroprevalencia de Brucella canis en perros callejeros del Centro de Bienestar Animal "La Perla", Medellín (Colombia), 2008. Revista Colombiana de Ciencias Pecuarias. 2010;23:166-72.

28. Tuemmers C, Lüders C, Rojas C, Serri M, Castillo C, Espinoza R. Detección de Brucella canis por método de inmunocromatografía en perros vagos capturados en la ciudad de Temuco, Chile, 2011. Rev Chil Infectol. 2013;30:395-401. https://doi.org/10.4067/S0716-10182013000400007

29. Hubbard K, Wang M, Smith DR. Seroprevalence of brucellosis in Mississippi shelter dogs. Prev Vet Med. 2018;159:82-6. https://doi.org/10.1016/j.prevetmed.2018.09.002

30. Chinyoka S, Dhliwayo S, Marabini L, Dutlow K, Matope G, Pfukenyi DM. Serological survey of Brucella canis in dogs in urban Harare and selected rural communities in Zimbabwe. J S Afr Vet Assoc. 2014;85:e1-e5. https://doi.org/10.4102/jsava.v85i1.1087

31. Hafemann DCM, Merlini LS, Gonçalves DD, Fortes MS, Navarro IT, Chiderolli RT, et al. Detection of anti-Leptospira spp., anti-Brucella spp., and anti-Toxoplasma gondii antibodies in stray dogs. J Venom Anim Toxins Incl Trop Dis. 2013;19:23-7. https://doi.org/10.1186/1678-9199-19-23

32. de Paula Dreer M, Gonçalves D, da Silva Caetano I, Gerônimo E, Menegas P, Bergo D, et al. Toxoplasmosis, leptospirosis and brucellosis in stray dogs housed at the shelter in Umuarama municipality, Paraná, Brazil. J Venom Anim Toxins Incl Trop Dis. 2013;19:23-7. https://doi.org/10.1186/1678-9199-19-23

33. Anigen Rapid C. Brucella Ab Test Kit. Fecha de consulta: 28 de septiembre de 2020. Disponible en: http://www.vetpetspharma.com/images/productos/canino/CBrucellaAb/ CBrucellaAb.pdf

34. Kim D, Park J, Kim SJ, Soh YM, Kim HM, Oh BH, et al. Brucella immunogenic BP26 forms a channel-like structure. J Mol Biol. 2013;425:1119-26. https://doi.org/10.1016/.j.jb.2013.01.015 
35. Sánchez-Jiménez MM, Ortiz-Román LF, Castrillón-Salazar LL, Giraldo-Echeverri CA, Olivera-Angel M. Application of a polymerase chain reaction test for the detection of Brucella canis from clinical samples of canines and humans. Revista Colombiana de Ciencias Pecuarias. 2014;27:3-11.

36. Olivera M, Giraldo CA, Di Lorenzo C. PCR identification of Brucella canis in canine blood and milk. A case report. Arch Med Vet. 2011;43:295-8. https://doi.org/10.4067/S0301-732X2011000300012.

37. Cárdenas D, Obando J, Moreno C, Mesa L, Ortiz A. Seroprevalencia de Brucella canis en la población canina del centro de zoonosis de la ciudad de Villavicencio. Revista Electrónica de Veterinaria. 2017;18:1-11.

38. Pardo A, Pérez C, Góngora A, Gómez L, Moreno A. Encuesta exploratoria de infección por Brucella canis en perros de Villavicencio-Colombia. Rev MVZ Córdoba. 2009;14:1690-6. https://doi.org/10.21897/rmvz.352

39. Agudelo-Flórez P, Castro B, Rojo-Ospina R, Henao-Villegas S. Seroprevalencia y factores de riesgo para brucelosis canina en perros domésticos de once comunas de la ciudad de Medellín-Colombia. Rev Salud Pública (Bogotá). 2012;14:644-56.

40. Castrillón-Salazar L, López-Diez L, Sánchez-Nodarse R, Sanabria-González W, HenaoCorrea E, Olivera-Angel M. Prevalencia de presentación de algunos agentes zoonóticos transmitidos por caninos y felinos en Medellín, Colombia. Rev MVZ Córdoba. 2019;24:711926. https://doi.org/10.21897/rmvz.1524

41. Ballut JC, Calderón A, Rodríguez V. Brucelosis en hembras caninas en Montería (Colombia): un problema para la salud pública. Biosalud. 2013;12:66-74.

42. Kaltungo BY, Saidu SNA, Sackey AKB, Kazeem HM. A review on diagnostic techniques for brucellosis. Afr J Biotechnol. 2014;13:1-10. https://doi.org/10.5897/AJB2013.13442

43. Carmichael LE, Shin SJ. Canine brucellosis: A diagnostician's dilemma. Semin Vet Med Surg Small Anim. 1996;11:161-5. https://doi.org/10.1016/s1096-2867(96)80028-4

44. Hollett RB. Canine brucellosis: Outbreaks and compliance. Theriogenology. 2006;66:575-87. https://doi.org/10.1016/j.theriogenology.2006.04.011

45. Greene CE, Carmichael LE. Canine brucellosis. En: Sykes J, Greene J, editors. Infectious Diseases of the Dog and Cat. $4^{\text {th }}$ edition. London: Elsevier Health Sciences; 2012. p. 398-411.

46. Cosford KL. Brucella canis: An update on research and clinical management. Can Vet J. 2018;59:74-81.

47. Ardoino SM, Baruta DA, Toso RE. Brucelosis canina. Ciencia Veterinaria. 2006;8:49-60.

48. Wanke M. Canine brucellosis. Anim Reprod Sci. 2004;82-83:195-207. https://doi.org/10.1016/j.anireprosci.2004.05.005

49. De Souza TD, De Carvalho TF, Mol JPDS, Lopes JVM, Silva MF, Da Paixão TA, et al. Tissue distribution and cell tropism of Brucella canis in naturally infected canine foetuses and neonates. Sci Rep. 2018;8:7203-12. https://doi.org/10.1038/s41598-018-25651-X

50. Aras Z, Uçan US. Detection of Brucella canis from inguinal lymph nodes of naturally infected dogs by PCR. Theriogenology. 2010;74:658-62. https://doi.org/10.1016/j.theriogenology.2010.03.023

51. Daly R, Willis KC, Wood J, Brown K, Brown D, Beguin-Strong T, et al. Seroprevalence of Brucella canis in dogs rescued from South Dakota Indian reservations, 2015-2019. Prev Vet Med. 2020;184:105157. https://doi.org/10.1016/j.prevetmed.2020.105157

52. Oosthuizen J, Oguttu JW, Etsebeth C, Gouws WF, Fasina FO. Risk factors associated with the occurrence of Brucella canis seropositivity in dogs within selected provinces of South Africa. J S Afr Vet Assoc. 2019;90:e1-e8. https://doi.org/10.4102/jsava.v90i0.1956

53. Lawaczeck E, Toporek J, Cwikla J, Mathison BA. Brucella canis in a HIV-infected patient. Zoonoses Public Health. 2011;58:150-2. https://doi.org/10.1111/j.1863-2378.2010.01334.x

54. Lucero NE, Maldonado PI, Kaufman S, Escobar GI, Boeri E, Jacob NR. Brucella canis causing infection in an HIV-infected patient. Vector Borne Zoonotic Dis. 2010;10:527-9. https://doi.org/10.1089/vbz.2009.0034

55. Dentinger CM, Jacob K, Lee LV, Méndez HA, Chotikanatis K, Mcdonough PL, et al. Human Brucella canis infection and subsequent laboratory exposures associated with a puppy, New York City, 2012. Zoonoses Public Health. 2015;62:407-14. https://doi.org/10.1111/zph.12163 
56. Lucero NE, Escobar GI, Ayala SM, Jacob N. Diagnosis of human brucellosis caused by Brucella canis. J Med Microbiol. 2005;54:457-61. https://doi.org/10.1099/jmm.0.45927-0

57. Tosi MF, Nelson TJ. Brucella canis infection in a 17-month-old child successfully treated with moxalactam. J Pediatr. 1982;101:725-7. https://doi.org/10.1016/s0022-3476(82)80301-6

58. Nomura A, Imaoka K, Imanishi H, Shimizu H, Nagura F, Maeda K, et al. Human Brucella canis infections diagnosed by blood culture. Emerg Infect Dis. 2010;16:1183-5. https://doi.org/10.3201/eid1607.090209

59. Swenson RM, Carmichael LE, Cundy KR. Human infection with Brucella canis. Ann Intern Med. 1972;76:435-8. https://doi.org/10.7326/0003-4819-76-3-435

60. Smith LM, Hartmann S, Munteanu AM, Villa PD, Quinnell RJ, Collins LM. The effectiveness of dog population management: A systematic review. Animals (Basel). 2019;9:1020-49. https://doi.org/10.3390/ani9121020

61. Maia C, Almeida B, Coimbra M, Fernandes MC, Cristóvão JM, Ramos C, et al. Bacterial and protozoal agents of canine vector-borne diseases in the blood of domestic and stray dogs from southern Portugal. Parasit Vectors. 2015;8:138-44. https://doi.org/10.1186/s13071-015-0759-8 